\title{
Spatial winding: cooperative heterogeneous multi-robot system for fibrous structures
}

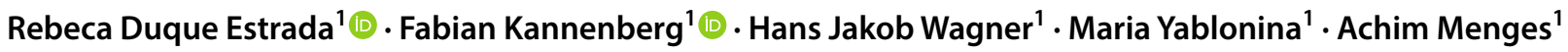

Received: 22 June 2020 / Accepted: 8 September 2020 / Published online: 21 September 2020

(c) The Author(s) 2020

\begin{abstract}
This research presents a cooperative heterogeneous multi-robot fabrication system for the spatial winding of filament materials. The system is based on the cooperation of a six-axis robotic arm and a customized $2+2$ axis CNC gantry system. Heterogeneous multi-robot cooperation allows to deploy the strategy of Spatial Winding: a new method of sequential spatial fiber arrangement, based on directly interlocking filament-filament connections, achieved through wrapping one filament around another. This strategy allows to create lightweight non-regular fibrous space frame structures. The new material system was explored through physical models and digital simulations prior to deployment with the proposed robotic fabrication process. An adaptable frame setup was developed which allows the fabrication of a variety of geometries within the same frame. By introducing a multi-step curing process that integrates with the adaptable frame, the iterative production of continuous large-scale spatial frame structures is possible. This makes the structure's scale agnostic of robotic reach and reduces the necessary formwork to the bare minimum. Through leveraging the capacities of two cooperating machines, the system allows to counteract some of their limitations. A flexible, dynamic and collaborative fabrication system is presented as a strategy to tailor the fiber in space and expand the design possibilities of lightweight fiber structures. The artifact of the proposed fabrication process is a direct expression of the material tectonics and the robotic fabrication system.
\end{abstract}

Keywords Robotic fabrication · Multi-robot system $\cdot$ Carbon fiber reinforced polymer $\cdot$ Long-span structures $\cdot$ Space frames $\cdot$ Spatial winding

\section{Introduction}

This research presents a cooperative heterogeneous multirobot fabrication system for the Spatial Winding of filament materials. The research focuses on the development of a robotic system that leverages the capacity of filament materials to be formed into space frame arrangements. This further expands the existing design space of filament structures in the context of architecture.

Rebeca Duque Estrada and Fabian Kannenberg contributed equally.

Fabian Kannenberg

fabian.kannenberg@icd.uni-stuttgart.de

Rebeca Duque Estrada

rebeca.duque@icd.uni-stuttgart.de

1 Institute for Computational Design and Construction, University of Stuttgart, Stuttgart, Germany

\subsection{Spatial structures}

The term space frame has been closely tied to large-span industrial buildings. Per definition, a space frame is "a structural system assembled of linear elements which are arranged so that the loads are transferred in a three-dimensional manner" (Elfawal 2014). These structures are minimal in their materialization, yet highly efficient and lightweight. With the Industrial Revolution the production of iron and then steel rapidly increased. These high-strength materials allowed to construct more lightweight structures with increased span and greater height (Chilton 2000). At the end of the 19th century Alexander Graham Bell developed the first prototypes of space frames and triangulated spaces as self-structuring systems, but they did not find their application in architecture until the introduction of the MERO system in 1943. The system developed by Dr. Ing. Max Mengeringhausen in Germany was the first space frame system widely available commercially (Chilton 2000). A conventional space frame structure consists of two-part types: node 
and edge. An edge is a linear element connecting two nodes. A node is an element that joins edges together into a spatial arrangement. Although nowadays traditional steel space frames can be customized, their main drawback is the inherent need for standardized parts as node designs can be highly complex. Specifically these joints can be very expensive, as they typically represent $30-50 \%$ of the total fabrication cost (SCI P358 2014) and require up to 50\% of the overall construction material (Narayanan 2006). This research proposes a continuous material approach to space frame structures, where the whole structure is made of a single continuous filament and the nodes are achieved through the wound fiberfiber connection (Figs. 1, 3). Applying the logic and rules of space frames to filament materials using robotic fabrication could enable the creation of lightweight, highly customizable structures at a variety of scales.

\subsection{Material system: lightweight fiber construction}

Due to their high load-bearing capacity and minimal selfweight, fiber-reinforced polymers (FRP) have been the preferred choice for structural applications in a variety of engineering fields, such as the automotive and aerospace industry (Prado et al. 2014). In these industries, fibers are typically wound or braided on full surface mandrels or complex pre-fabricated molds (Fig. 2). As architecture rarely goes into serial production and almost every project is a unique structure, complex formwork often defies the economical applicability of FRP in architectural projects. Still,
FRP also show great potential for strong and lightweight structures in architecture (Reichert et al. 2014; Doerstelmann et al. 2015; Vasey et al. 2015); (Felbrich et al. 2017; Bodea et al. 2020; Kayser et al. 2019). In particular carbon fiber reinforced polymers (CFRP) can reach impressive tensile strength, much higher than steel wires, while their densities are much lower (Liu et al. 2015). Their anisotropy and low weight make them excellent material for high-performance structures with programmed directionality. The current state of the art in Coreless Filament Winding (CFW), specifically in architectural research - demonstrates how a full surface mandrel can be reduced to anchor points arranged on a linear scaffolding (Menges 2016). The Fibre Facade project demonstrates a different approach in which the general concept behind CFW is applied to a truss-type structure (Solly 2020). The structural capacity of CFRP is visually demonstrated by using a minimal amount of material to configure the final supports of the facade panels (Solly 2020).

\subsection{Multi-robot fabrication}

Multi-robot fabrication methods allow for complex fabrication tasks through cooperative routines (Alvarez et al. 2019). Multi-robot systems can be categorized as homogeneous and heterogeneous robot teams. Just as it is easier for humans to produce something with two hands rather than only one, homogeneous cooperation of two robotic arms can drastically increase the achieved dexterity (Parascho et al. 2017). On the other hand, there have been

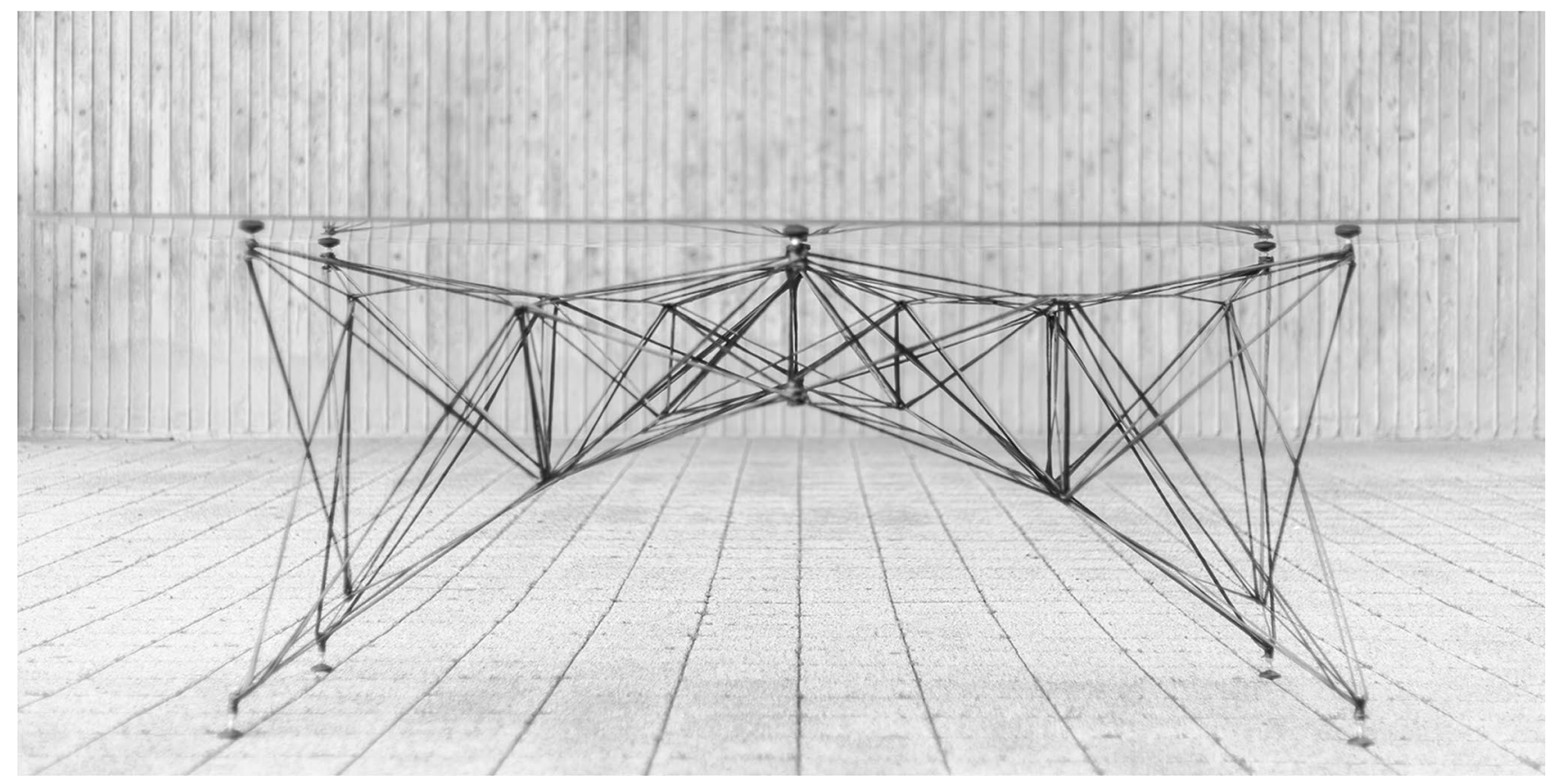

Fig. 1 Demonstrator one: Spatially wound table (size: 1.6 x 0.8 x $0.75 \mathrm{~m}$ ) 

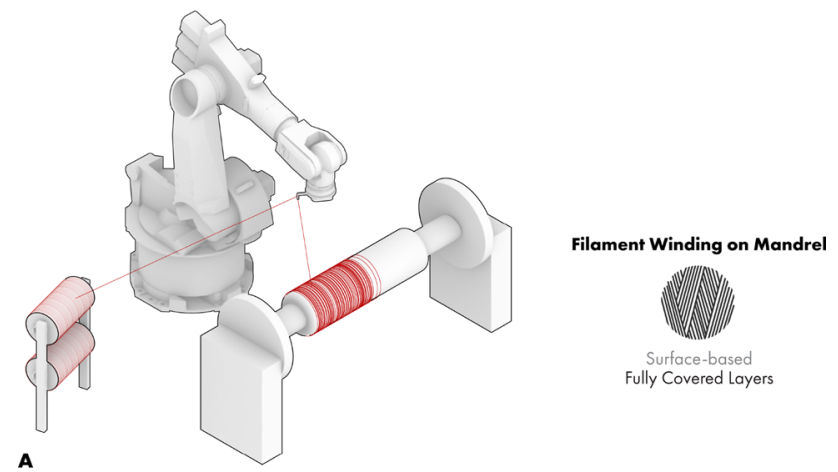

A
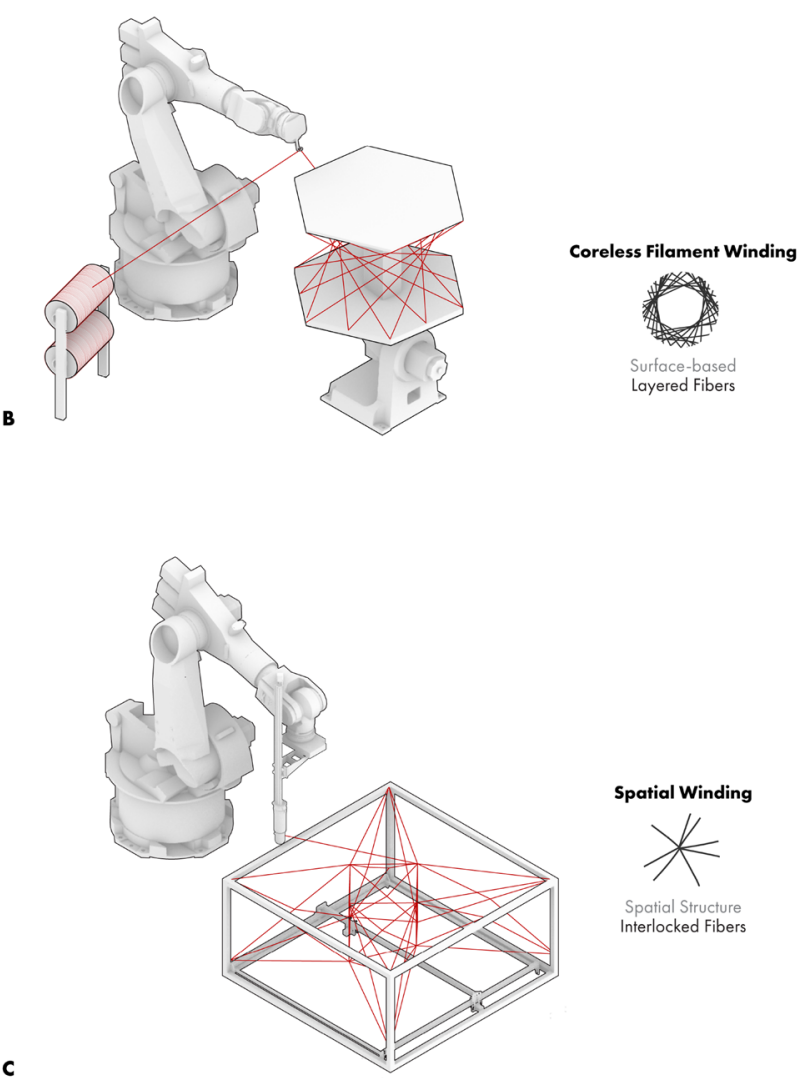

Spatial Structure Interlocked Fibers

Fig. 2 Comparison of filament winding methods: a Filament winding on mandrel, b coreless filament winding, $\mathbf{c}$ spatial Winding

successful examples for heterogeneous teams of bespoke mobile robots that are very task-specific and essentially able to walk over, under or in between the structure. The increased complexity of fabrication goals can be divided into an array of simpler tasks that can then be distributed to single-task construction robots (Bock and Linner 2016). Bespoke mobile robots could augment or potentially replace off-the-shelf industrial machines (Yablonina and Menges 2018). These machines could work individually but also cooperatively. Such heterogeneous cooperation can be seen in the Minibuilders project by Jokic et al.

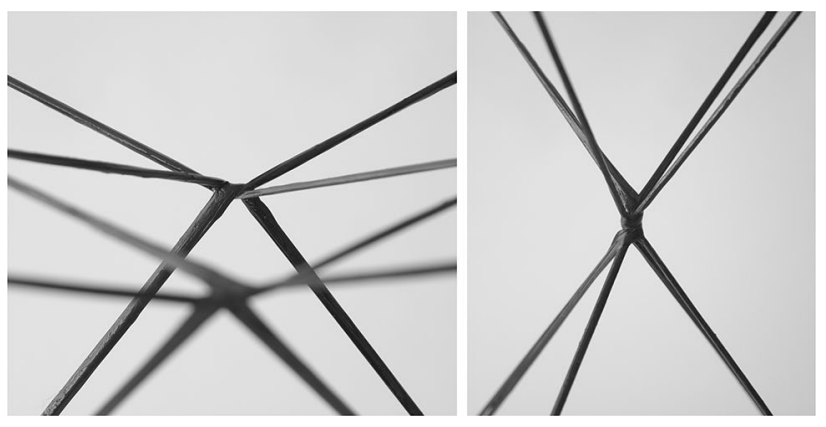

Fig. 3 Spatial winding: details of fiber-to-fiber interaction creating nodes in space

(2014) where each machine is building upon the previous machine's step.

Some projects have demonstrated the potential of multiple machines cooperating using filament materials. Mirjan et al. (2013) present an aerial construction process in which tensile structures are autonomously built with unmanned aerial vehicles (UAV). Choreographed movements of the multiple machines cooperating in the assembly task enables new material arrangements. Since the spool is directly attached to the quadcopters, they are able to fly through and weave between the existing ropes and essentially use part of the structure as formwork for the next part. By precisely calculated maneuvers, the quadcopters are able to assemble new fiber nodes in space. Except for the required anchor points at both ends of the structure, the demonstrators' connections and links are entirely realized by the flying machines (Augugliaro et al. 2015).

The ICD/ITKE 2016/17 Research Pavilion demonstrates how heterogeneous multi-machine cooperation can be used to hand off material from one machine to another and therefore extend the reach of an industrial robot. It combines low payload yet long-range machines, such as UAVs with strong, precise but limited-reach industrial robots. This multimachine cooperation allowed the scalable fabrication setup of a long-span FRP structure. The novel robotically driven manufacturing process enabled a highly differentiated continuous fiber structure (Felbrich et al. 2017). The presented research aims to develop a novel bottom-up design logic for fiber-reinforced structures through the development of a heterogeneous multi-robot fabrication system that reduces the required formwork to a minimum while opening up new geometrical possibilities.

\section{Methods}

This research follows an integrated co-design methodology, in which geometry and material characteristics are tightly integrated with novel robotic fabrication techniques. The 
three topics were explored and developed through physical and digital means. This paper focuses specifically on the development of the heterogeneous multi-robot fabrication system which allowed the fabrication of a long-spanning lightweight spatial structure through the introduction of fiber-fiber wound nodes. The methodology regarding the geometrical system and the computational tools will be briefly explained, while the methods for hardware and software in the development of the multi-robot system receive greater attention.

\subsection{Spatial winding}

Coreless Filament Winding (CFW) methods typically use bespoke steel frames and are used to wind surface-based geometric typologies (Prado et al. 2014). In these cases, the interaction between fibers happens as an additive process, stacking layer after layer (Fig. 2). Although this enables the generation of a covered surface, a relatively big amount of fibers and anchor points are necessary, and the frame can become overwhelmingly complex.

The term Spatial Winding is introduced here as a new method for fabricating lightweight spatial structures. While CFW is based on winding the fiber around anchor points, Spatial Winding combines winding around anchors and winding around the fiber itself. In order to allow this method, the material is removed from its usually stationary condition, being able to freely move in space. Weaving, as a textile production technique, introduced the concept of warp and weft turning yarn into fabric. In a three-dimensional way, a similar principle of over-and-under motion is combined within the proposed method assuring proper fiber interaction (Fig. 3). A new set of syntax logics and geometric rules were developed, informed by both the fabrication process and the material system, enabling the creation of a catalog of diverse geometries within the same generic frame (Fig. 4) (Duque Estrada et al. 2020).

The principles were established on a minimal setup (four to eight anchors), setting the fundamental criteria to form a geometry. Through diverse iterations of empiric physical experiments, a range of material behaviors were observed and transformed into syntax logics, creating a rich set of parameters to be tuned to define the final geometry. For the physical experiments both off-the-shelf and custom-made pre-impregnated carbon fibers were tested.

Spatial Winding expands on the methodology of Coreless Filament Winding, further disentangling the direct dependence between frame and object geometry. It extrapolates the boundaries of robotic fabrication and proposes a new set of geometrical rules for large-scale fibrous structures. This method enables the fiber to work as formwork for other fibers, resulting in the use of fewer anchors, therefore a simpler frame. Even with a simpler frame, the generated
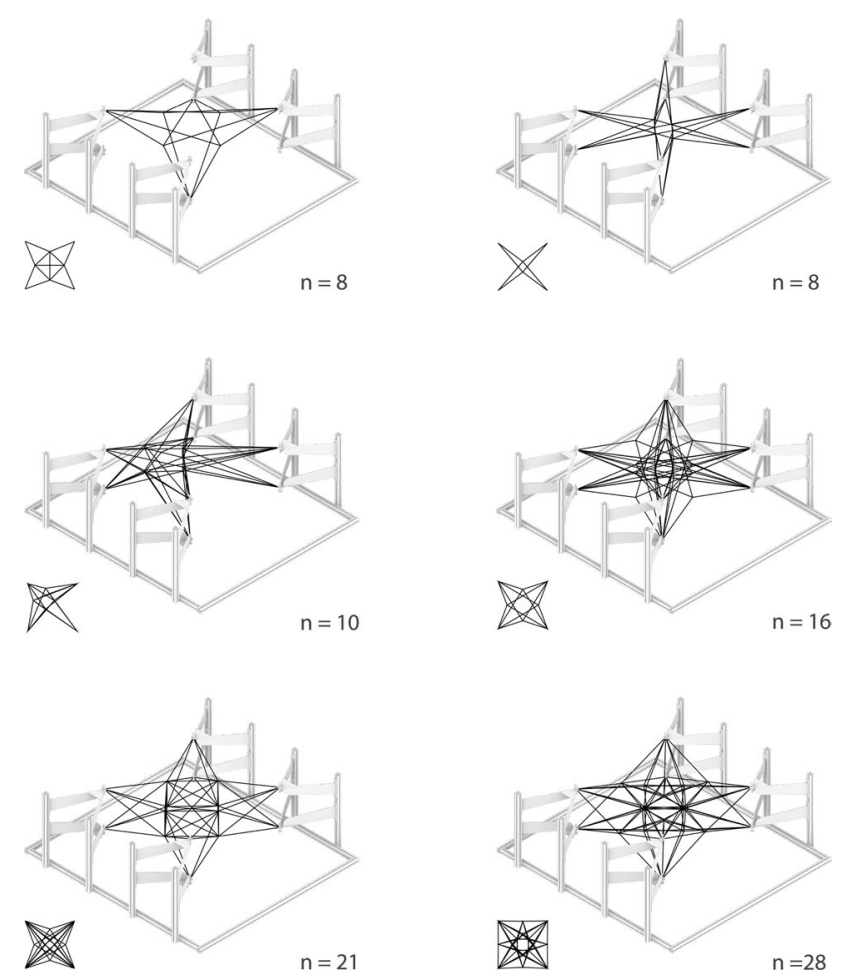

Fig. 4 Different geometries can be generated within the same generic frame. $n$ shows the number of additional nodes in space created by the syntax

geometries can achieve a high level of complexity, which requires the use of computational tools to mediate design and materialization.

\subsection{Digital tools}

A digital form-finding tool was developed to assist the design process and rapidly iterate through possible geometries. The design tool was created using spring based relaxation with the live physics engine Kangaroo (Piker 2013). The syntax was defined as straight lines between anchor points and node subdivisions. By relaxing those lines toward target points, different geometries were generated and informed the rest lengths of the individual edges for future fabrication. Taking the structural qualities of CFRP into account, in particular, its high tensile strength, it was important to inform the design decisions with the material properties. A structural optimization with Finite Element Analysis (FEA) was used to simulate the model under load and define the optimal cross-section of the fiber segments (Duque Estrada et al. 2020). By giving real-time feedback on how syntax strategies would interfere with the distribution of forces, the digital simulation allowed for a better understanding of the relationship between geometry, material, and structural behavior. 


\subsection{Multi-robot cooperation}

The fundamental aspect for multi-robot cooperation (MRC) in this project stems from the need of having two agents working on opposite sides of a workpiece. MRC enables material manipulation with a free end, combining different tasks such as unspooling the material, winding around anchors and exchanging the spool (Fig. 9). By placing the material spool directly on the end effector, the system allows to wrap the fiber around other fibers in the workspace enabling an interlocked fiber interaction, which is the core concept of the proposed geometrical system (Fig. 3). Two crucial robotic fabrication routines were established: winding and material exchange. The routines were distributed between two robotic agents as follows: the winding agent performs the winding around the anchors and most of the material transportation; the material exchange agent receives and delivers the material, allowing the spool to pass under or around the already wound fibers. Given the need to apply a high force during winding, to ensure the tension on the material, a 6-axis robotic arm was chosen as the winding agent. Taking into account the importance of precision and speed required for the material exchange agent, a CNC gantry was defined (Fig. 5a). Both agents received customized end-effectors that increased the final dexterity of the robotic system. A variety of spatial relationships between the robotic agents were identified: Vertical exchange, lateral exchange and angular exchange. While the lateral exchange presents advantages regarding the geometry of the fabricated artifact, it implied a level of unnecessary complexity of the robotic maneuvering and control. Within the scope of this project, the vertical and angular exchanges were identified as the most promising and thus have been investigated further in the demonstrator.

\section{Development}

\subsection{System overview}

The fabrication setup is composed of two active agents and an adaptable frame (Fig. 5a). The frame is located between the agents and consists of a simple stiff structure to hold the few anchor points in place (Fig. 5b). The position of each anchor can be adjusted, enabling the fabrication of elements with different boundary conditions. Both agents were equipped with customized end-effectors including the necessary mechanisms to perform specific tasks, augmenting their abilities and reachability (see Sect. 3.3). Through the research, two demonstrators were built. A spatially wound table (Fig. 1) was a proof of concept of the proposed material system and was used to fine-tune the
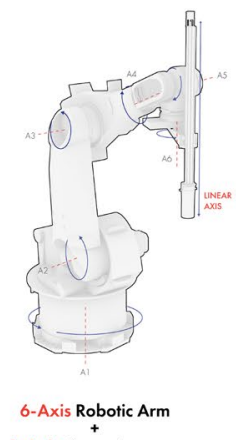

1-Axis Linear Actuator

A

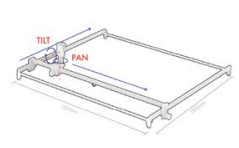

2-Axis CNC Gantry 2-Axis Tilt-Pan Effector

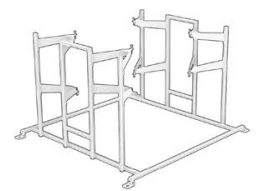

8 Anchors Reconfigurable Frame Aluminum Profiles + Steel Plates
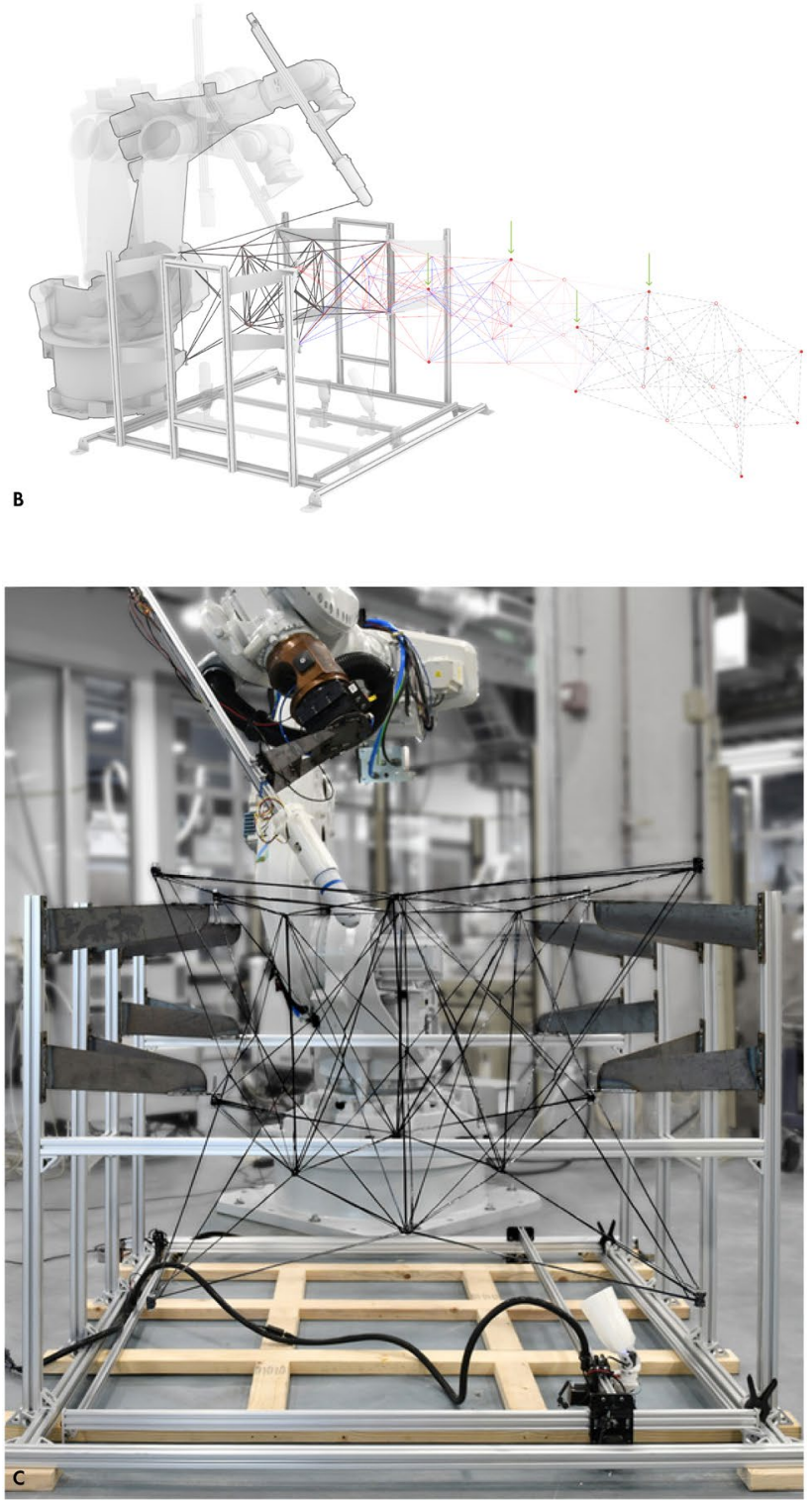

Fig. 5 a Fabrication agents with custom end-effectors and reconfigurable frame, $\mathbf{b}$ complete multi-robot fabrication setup showing winding of an element, $\mathbf{c}$ multi-robot fabrication setup for demonstrator two 
simulation and optimization tool. The second demonstrator, a spatially wound long-span structure $(4 \times 0.9 \times 0.7 \mathrm{~m})$ was designed and built to validate the robotic fabrication routines (Figs. 5c, 10). The dimensions of both demonstrators were constrained by the size of the curing oven (see Sect. 3.4).

\subsection{Design to fabrication workflow}

The workflow begins with a design loop, in which global design intentions, boundary conditions, and syntax logic inform each other giving form to an initial geometry (Fig. 6). Furthermore, the global geometry can be connected to the form-finding tool, with which it is possible to iterate through different shapes by moving the position of some of the spatial nodes. Once the geometry is satisfactory, it is transformed into a parametric model that enables the control of the boundary condition. At this point, the ranges of displacement for each anchor and spatial nodes are set and will define how much the structure can change during the optimization process. Structural results from the FEA are connected to the evolutionary solver Galapagos (Rutten 2013) that optimizes the cross-sections of the fiber segments as well as the placement of anchor points within the design intention. The solution with the best fitness is then used to generate the motion paths for both agents. Custom offline path planning methods were developed for the material exchange and anchoring routines, which allowed to adapt the robot paths to different geometries directly from the digital model. The robotic arm receives the RAPID code (highlevel programming language used to control $\mathrm{ABB}$ industrial robots) for the material exchange positions and anchoring routines and the CNC gantry receives the G-Code for the material exchange positions. Both end-effectors receive their task-specific commands as a serial message.

\subsection{End-effectors}

Bespoke end-effectors were developed for both robotic agents to perform the actions needed to complete the fabrication process (Fig. 7). To avoid unwanted unspooling and facilitate the material exchange, the material is stored in spools protected by a case and, when necessary, can be changed for new ones (Fig. 7b).

Winding Effector (on Robot) The robot end-effector has three tasks: hold the spool case, lock the spool rotation and extend itself to grab and pass the spool case through the fibers to the $\mathrm{CNC}$ gantry effector. For the first goal, an electropermanent magnet (EPM) was placed inside the cup holder and connected to a microcontroller. After activated the EPM creates a magnetic field until the next activation, presenting a good solution to avoid overheating and constant power consumption of regular electromagnets (Knaian 2010). The EPM's payload is about $15 \mathrm{~kg}$ and can be controlled with $5 \mathrm{~V}$. The locking mechanism was designed to stop the spool rotation, enabling the robot to selectively apply tension on the fiber

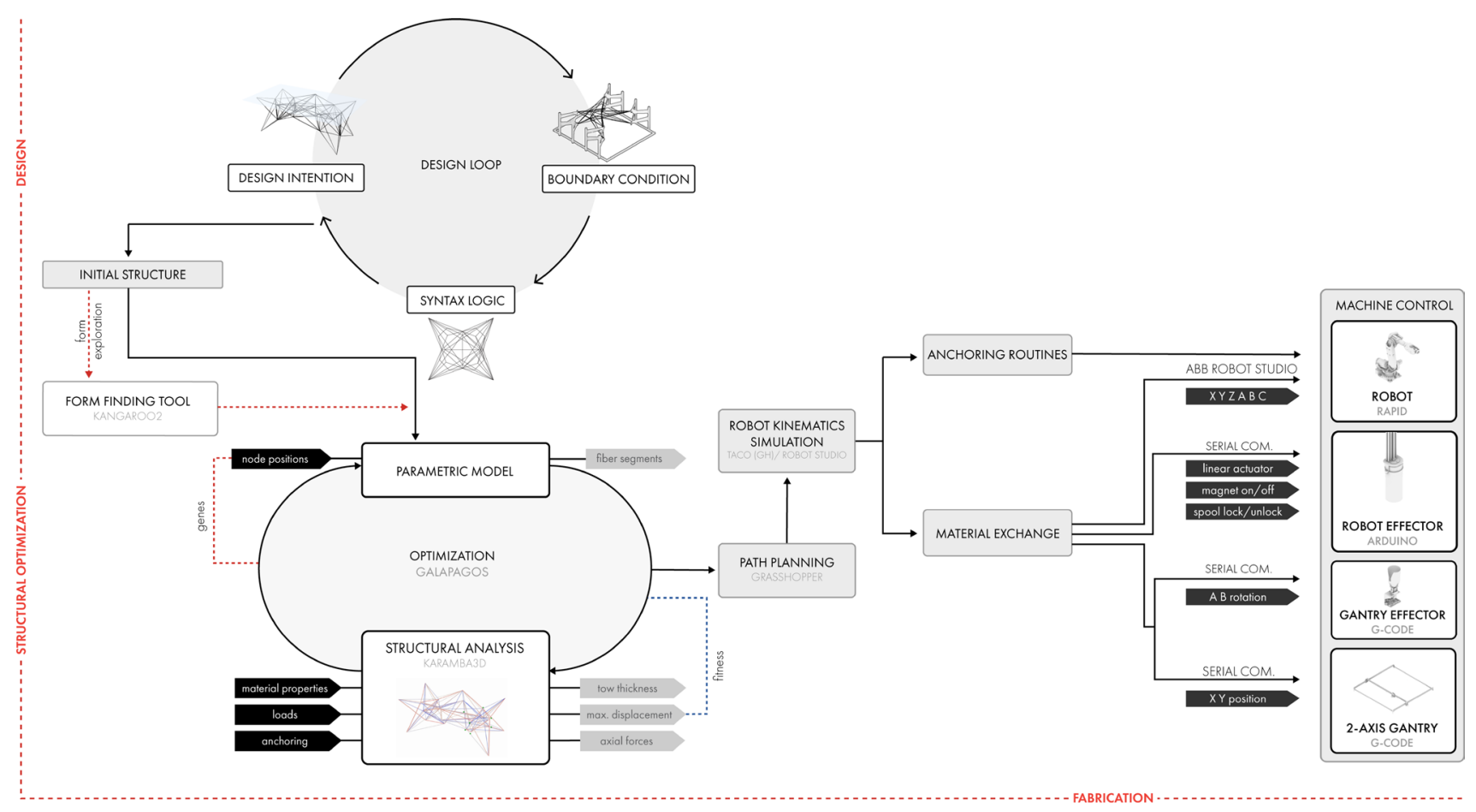

Fig. 6 Design to fabrication workflow 
Fig. 7 Exploded isometric of the custom end-effectors: a Winding (robot) effector, $\mathbf{b}$ material bobbin, $\mathbf{c}$ material transport (CNC gantry) effector

during winding. Different strategies were tested and a servo with a double spur gear mechanism and metal pins on a rack presented to be the best solution. By going halfway down, the metal pins can lock the case rotation and when going fully down, it locks the spool rotation as well. An off-theshelf linear actuator of one meter was installed on the adaptor attached to the robot's flange. It was possible to use the linear actuator to extend the reach of the robot enabling the effector to approach the CNC gantry and exchange the spool with the material through the fibers (Fig. 7a).

Material Transport Effector (on CNC Gantry) The task of the CNC gantry end-effector was simpler. It needed to receive and give the spool case at a specific angle when necessary. Two servos with position feedback and high torque were used to assemble a pan and tilt mechanism, adding two more degrees of freedom to the 2-axis CNC gantry (the $\mathrm{Z}$-axis was not installed). This way the CNC gantry was able to move the effector around and for complex angular material exchanges, it would rotate to adjust the cup's position (Fig. 7c).

Machine Control and Communication In conventional path planning for CFW, the robot winds each fiber segment by connecting anchors. In contrast, our path planning method consists of a set of planes in space for the exchange of material for both robotic arm and CNC machine. For the anchoring routines, the paths are created considering the best orientation for the robot tool center point in relation to the anchor, trying to avoid stress on the material while unspooling. The positions for the exchange of material were synchronized for both the robotic arm and CNC gantry and the angles for specific exchanges were defined. A touch up of the winding anchors allowed to calibrate the CAD data with the physical setup and calibrate both the coordinate systems of the robot and the $\mathrm{CNC}$ gantry. The $\mathrm{CNC}$ gantry received G-code consisting of $\mathrm{X}$ and $\mathrm{Y}$ coordinates as well as $\mathrm{A}$ and $\mathrm{B}$ rotations for the additional degrees of freedom of the end-effector. A custom tool was developed to control and visualize its current state from within Grasshopper. The robot's bespoke end-effector receives signals to position the linear actuator to reach through the fibers, activates or deactivates the EPM to pick up the spool and lock the spool rotation with a servogear-mechanism (Fig. 9a).

\subsection{Multi-step curing}

In contrast to typical manufacturing methods based on the assembly of discrete elements with distinct functions, this
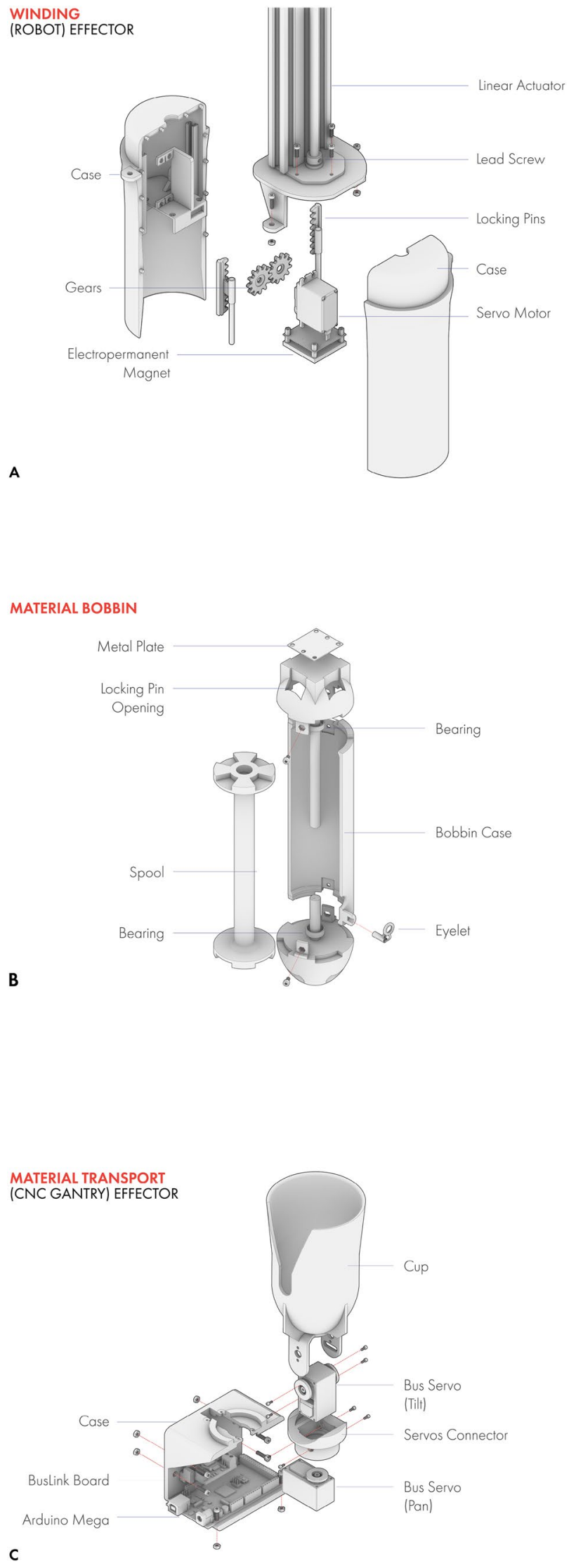

C 
research proposes a hybrid between component-based and continuous systems, in which the fabrication method enables the structure to grow through a multi-step process and tries to benefit from both systems. The hybrid system happens through a multi-step curing process, in which the first element is fabricated and after cured, it changes its position on the frame, shifting the attachment of the anchor points, enabling the next element to be fabricated on the same previous anchors (Fig. 8). This way, the structure can grow in different directions sharing the same anchors and avoiding weak mechanical connections. By merging both systems, it is possible to keep the frame small and simple and stay within the regular reach of the robot, removing the need for external axes or tracks. The frame sets the boundary condition and size of each element produced, but it does not directly refer to the size and complexity of the final structure. However, the dimensions of the curing oven will constrain its final size. For each element, the amount of nodes and connections inside the boundary is not immediately related to the number of anchors. The multi-step curing process and the multi-robot fabrication system were proven with demonstrator two (Fig. 10). It was possible to re-anchor already cured elements to the frame and interweave it with the new element (Fig. 8b). The structure was able to support itself and cantilever out of the frame, even for the last, fourth element no additional support was needed during curing.
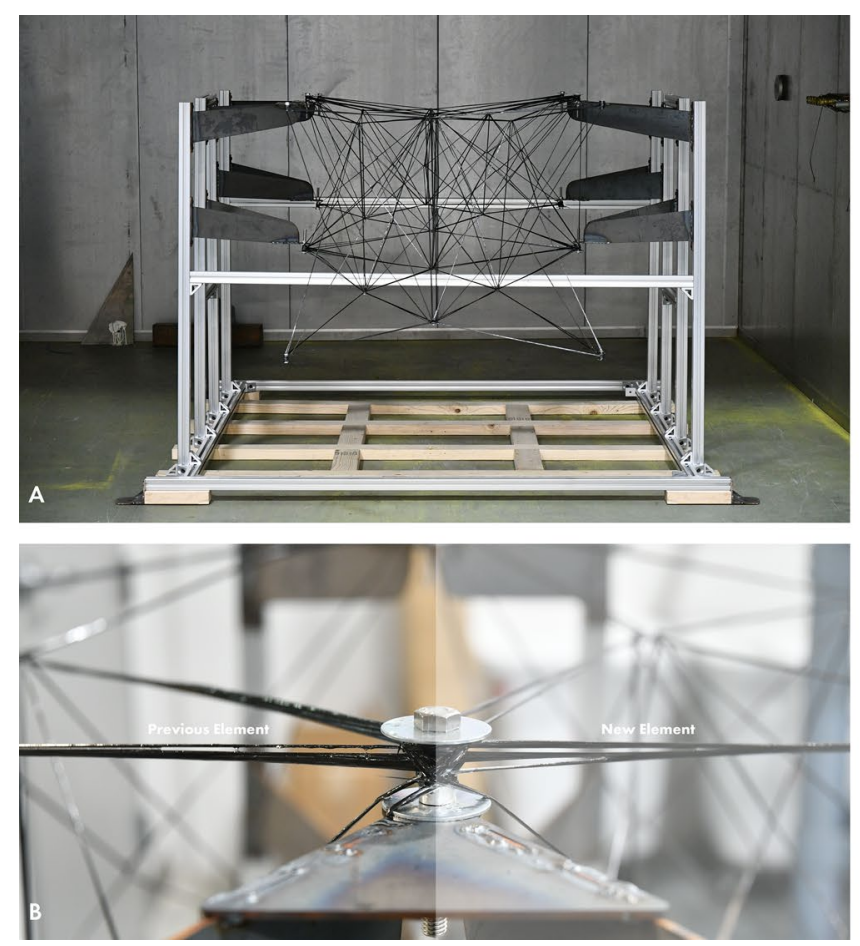

\section{Discussion}

The work presented here introduces a novel methodology integrating computational design, digital fabrication and material performance. Through Spatial Winding, it was demonstrated how two non-identical machines can cooperatively wind, weave and exchange material, enabling the fabrication of large-scale, non-uniform spatial structures. Two prototypical demonstrators exemplify the potentials for such lightweight structures. Nonetheless, some aspects of the research would require further technical development which will be discussed in this section.

\subsection{System potentials and limitations}

Apart from the structural potential as a load-path optimized space frame, the Spatial Winding system implies aesthetic potentials such as density tailoring and placement and sizing of apertures. The system's potential of achieving a wide range of varying geometries within a simple generic frame is to be further explored in future research. It will require a more detailed investigation of frame hardware and geometry, towards more configurable boundary conditions. The proposed hybrid system with the multi-step curing process allows to build long-span structures agnostic of the limited robot reach. However, the size of the structure is currently

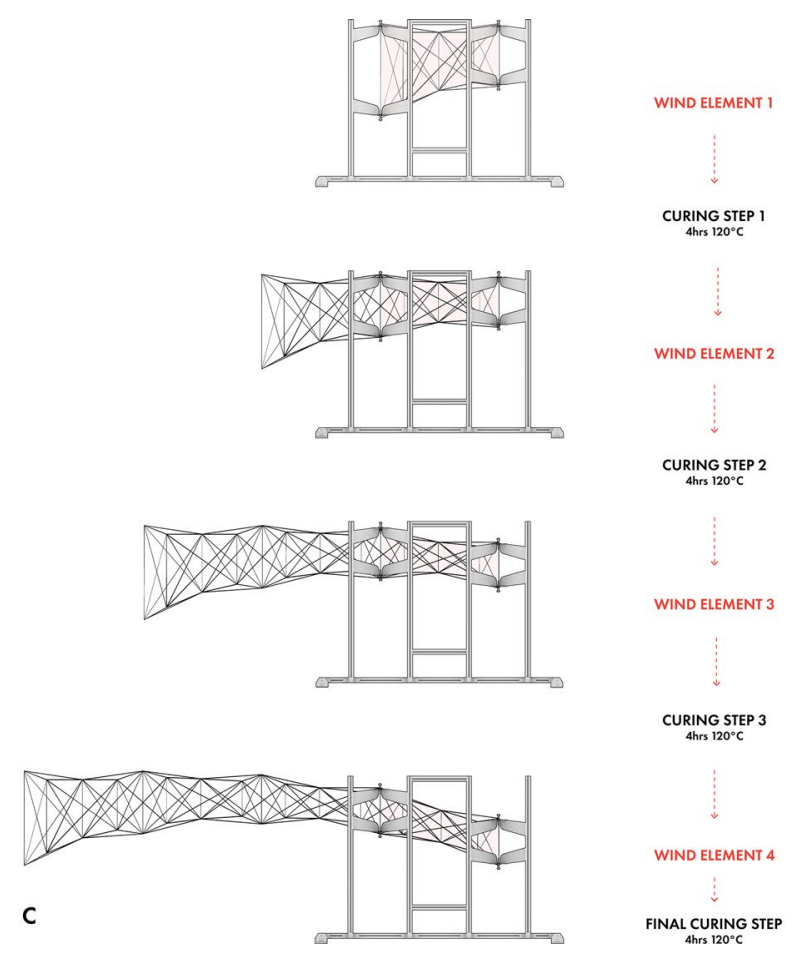

Fig. 8 a Frame with two wound elements in curing oven, b Interface between previous (cured) element and newly wound element, c Multi-step curing schematic 

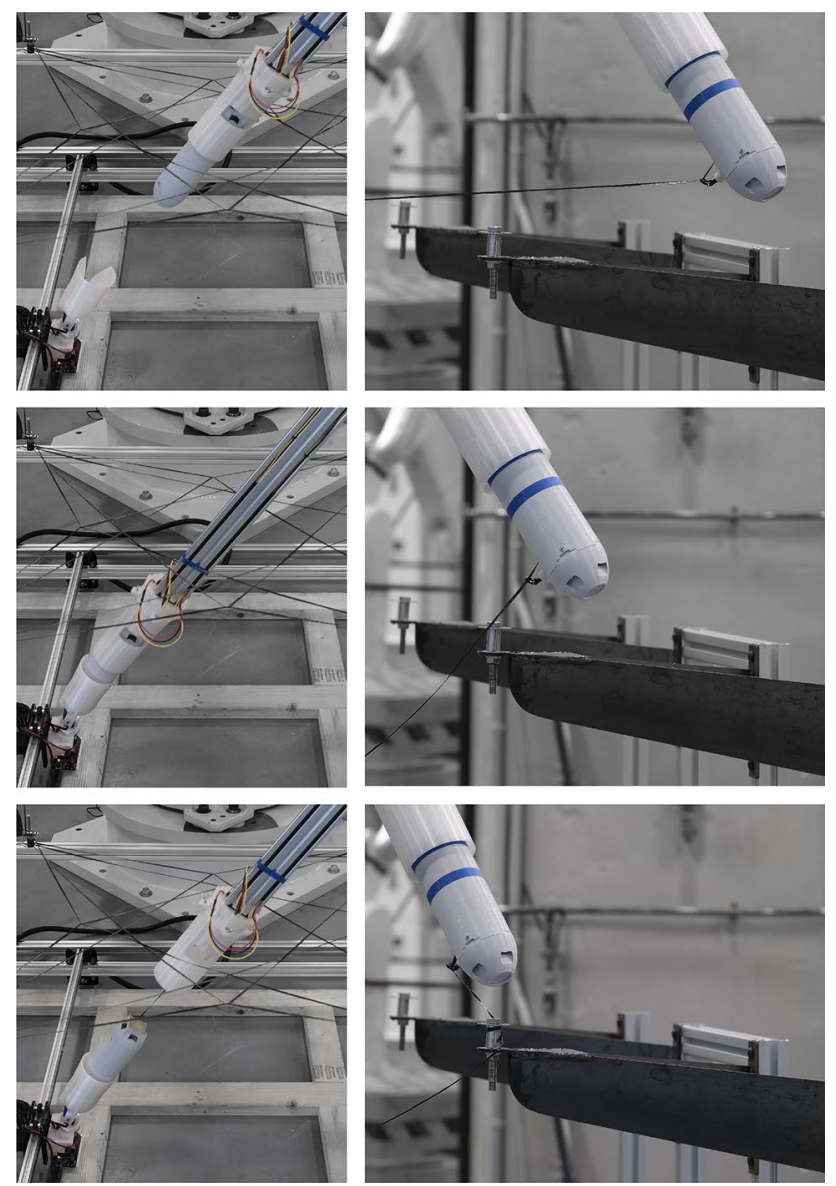

Fig. 9 a Material exchange routine (left), b unspooling extra material to create desired length of slack and winding around anchor (right)

dependent on the size of the curing oven. A different curing solution, such as electrical curing for carbon (Sarles et al. 2006) or UV curing for glass fibers (Kayser et al. 2019) could potentially be used to bypass those limitations.

\subsection{Further development}

The robotic path planning in a highly constrained threedimensional space is a challenging task (Fig. 9a). The inverse kinematics solver had to be continuously surveyed to avoid collisions with either frame or fibers and each new element that was fabricated required adjustments to the path planning algorithm. A possible solution for a fabrication environment that changes over time could be the integration of live sensor data. Such a cyber-physical system allows constant feedback between the actual fabrication environment and the digital generation of robot control codes in real-time (Vasey et al. 2015). Surveying methods such as optical 3D scanners or LIDAR (Light Detection and Ranging) could potentially be used to iteratively record data of the physical structure. Changing the directionality of the fibers is usually considered to be detrimental to the fiber's performance (Knippers et al. 2011). As this is inherent to the spatially wound nodes, further research should be carried out by structural testing of the fiber-fiber nodes.

\section{Conclusion and outlook}

The fabrication of the final demonstrator (Fig. 10) represented a crucial moment in the research as all areas of development came together in one integrated system. The multirobot system is not limited to two agents. It could reach a much higher degree of complexity when other machines are added to it. Even a second fiber bobbin could be considered to create temporary nodes in space without the immediate need for anchoring. Scale is an interesting factor for this research, in terms of resolution as well as physical dimensions. The proposed system is situated in a lab environment but could be made more robust and taken on site. This would allow the continuous structure enabled by the multi-step curing process to bypass size limitations of transportation or fabrication space. The proposed system, where both members and nodes are of the same continuous material could be a solution to the usually highly expensive node fabrication in traditional space frame structures. By creating a robotic system in parallel to the material system a reciprocal relationship between material, technology and design emerges, expanding the boundaries of known systems and design possibilities for robotically fabricated lightweight structures. 


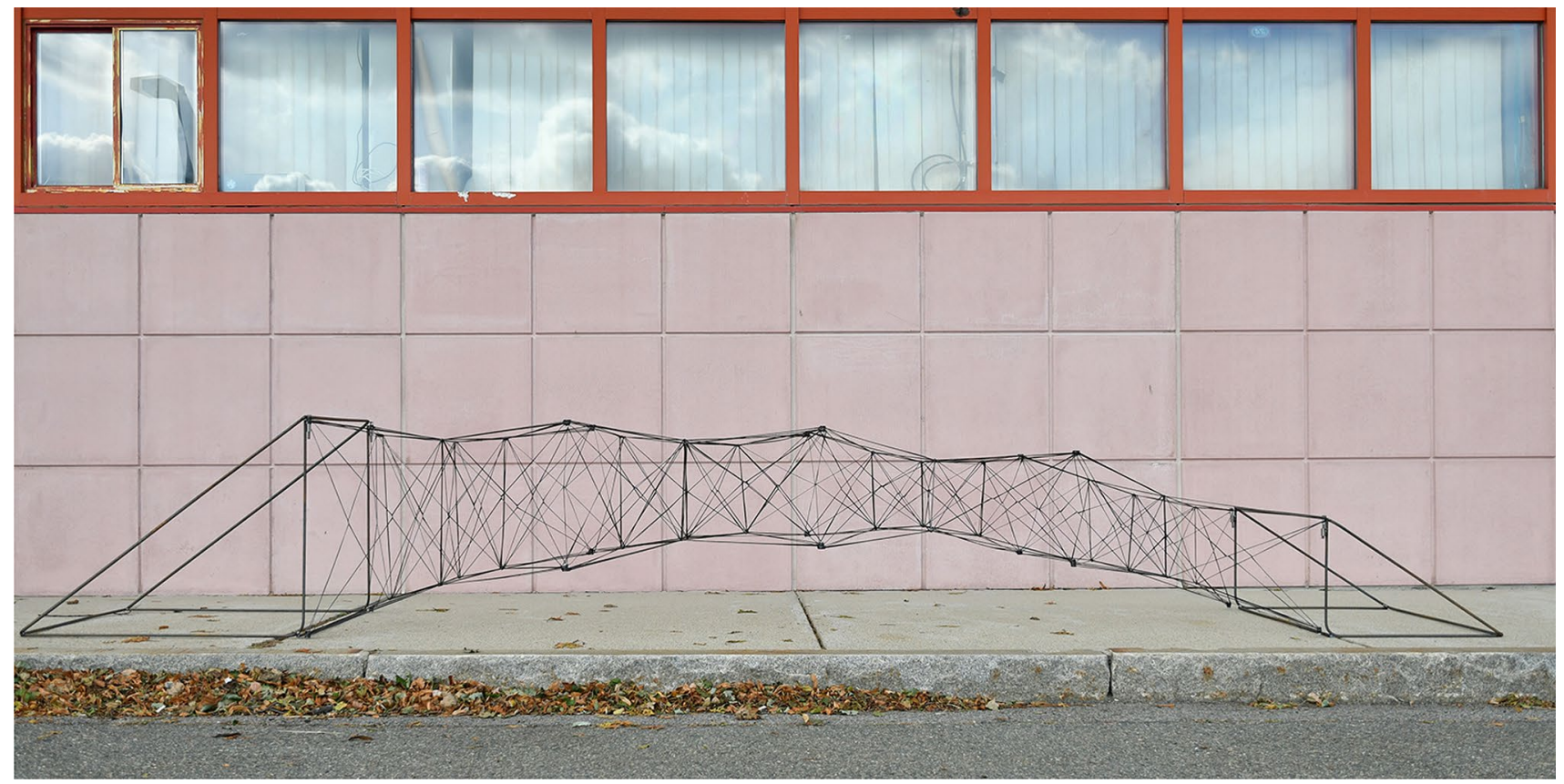

Fig. 10 Demonstrator two: Spatially wound long-span structure (size: 4 x 0.9 x 0.7 m)

\begin{abstract}
Acknowledgements The research was partially supported by the German Research Foundation under Germany's Excellence Strategy - EXC 2120/1 - 390831618. The project was developed by the first authors within the ITECH MSc program "Integrative Technologies and Architectural Design Research" offered by ICD and ITKE at Stuttgart University. The authors would like to thank Autodesk, OpenBuilds, TCR Composites and FibR GmbH for their support. The final demonstrator was fabricated within a two months residency at the Autodesk Technology Center Boston.
\end{abstract}

Funding Open Access funding enabled and organized by Projekt DEAL. The research was partially supported by the German Research Foundation under Germany's Excellence Strategy - EXC 2120/1 - 390831618.

\section{Compliance with ethical standards}

Conflict of interest The authors declare that they have no conflict of interest.

Open Access This article is licensed under a Creative Commons Attribution 4.0 International License, which permits use, sharing, adaptation, distribution and reproduction in any medium or format, as long as you give appropriate credit to the original author(s) and the source, provide a link to the Creative Commons licence, and indicate if changes were made. The images or other third party material in this article are included in the article's Creative Commons licence, unless indicated otherwise in a credit line to the material. If material is not included in the article's Creative Commons licence and your intended use is not permitted by statutory regulation or exceeds the permitted use, you will need to obtain permission directly from the copyright holder. To view a copy of this licence, visit http://creativecommons.org/licenses/by/4.0/.

\section{References}

Alvarez M, Wagner HJ, Groenewolt A, Krieg OD, Kyjanek O, Sonntag D, Bechert S, Aldinger L, Menges A, Knippers J (2019) The buga wood pavilion. In: ACADIA 19: Ubiquity and AutonomyProceedings of the 39th Annual Conference of the Association for Computer Aided Design in Architecture (ACADIA), The University of Texas at Austin School of Architecture, Austin, Texas, Acadia Publishing Company, pp. 490-499

Augugliaro F, Zarfati E, Mirjan A, D’Andrea R (2015) Knot-tying with flying machines for aerial construction. In: 2015 IEEE/ RSJ International Conference on Intelligent Robots and Systems (IROS), pp. 5917-5922

Bock T, Linner T (2016) Construction robots: elementary technologies and single-task construction robots, vol 3. Cambridge University Press, Cambridge

Bodea S, Dambrosio N, Zechmeister C, Gil Pérez M, Koslowski V, Rongen B, Dörstelmann M, Kyjanek O, Knippers J, Menges A (2020) BUGA fibre pavilion: towards robotically-fabricated composite building structures. UCL Press, London, pp 234-243

Chilton J (2000) Space grid structures. Architectural Press, New York

Doerstelmann M, Knippers J, Menges A, Parascho S, Prado M, Schwinn T (2015) Icd/itke research pavilion 2013-14: modular coreless filament winding based on beetle elytra. Archit Des 85(5):54-59

Duque Estrada R, Kannenberg F, Wagner HJ, Yablonina M, Menges A (2020) Integrative design methods for spatial winding. In: Baverel O, Mueller C, Pottman H, Tachi T (eds) AAG 2020, advances in architectural geometry. Klein Publishing $\mathrm{GmbH}$, École des Ponts ParisTech, Paris

Elfawal MFA (2014) The technical and feasibility selection of space truss construction systems. Mobile and rapidly assembled structures IV. IT Press, Southampton, pp 293-303 
Felbrich B, Frueh N, Prado M, Saffarian S, Solly J, Vasey L, Knippers J, Menges A (2017) Multi-machine fabrication: An integrative design process utilising an autonomous uav and industrial robots for the fabrication of long span composite structures. In T. Nagakura (Ed.), ACADIA - Disciplines \& Disruption, Proceedings of the ACADIA Conference 2017, US, pp. 248-259. Acadia Publishing Company

Jokic S, Novikov P, Maggs S, Sadan D, Jin S, Nan C (2014) Robotic positioning device for three-dimensional printing. CoRR abs/1406.3400. https://arxiv.org/abs/1406.3400

Kayser M, Cai L, Bader C, Falcone S, Inglessis N, Darweesh B, Costa J, Oxman N (2019) Fiberbots: design and digital fabrication of tubular structures using robot swarms. Robot Fabr Archit Art Des 2018:285-296

Knaian AN (2010) Electropermanent magnetic connectors and actuators: devices and their application in programmable matter. Massachusetts Institute of Technology, USA

Knippers J, Cremers J, Gabler M, Lienhard J (2011) Construction manual for polymers + membranes. https://www.researchga te.net/publication/264082967_Construction_Manual_for_Polym ers_Membranes

Liu Y, Zwingmann B, Schlaich M (2015) Carbon fiber reinforced polymer for cable structures-a review. Polymers 7:2078-2099

Menges A (ed) (2016) Material performance: fibrous tectonics and architectural morphology (Harvard GSD Studio Reports). Harvard GSD studio reports. Harvard University Graduate School of Design, Cambridge

Mirjan A, Gramazio F, Kohler M, Augugliaro F, D’Andrea R (2013) Architectural fabrication of tensile structures with flying machines. In: Ferreira T (ed) Green design, materials and manufacturing processes. CRC Press, Hoboken, pp 513-518 SIM2013 - International Conference on Sustainable Intelligent Manufacturing; Conference Location: Lisbon, Portugal; Conference Date: June 26-29, 2013

Narayanan S (2006) Space structures: principles and practice in space structures. Multi-Science Publishing, London

Parascho S, Gandia A, Mirjan A, Gramazio F, Kohler M (2017) Cooperative fabrication of spatial metal structures. In: Menges A, Sheil B, Glynn R, Skavara M (eds) Fabricate. UCL Press, Stuttgart, pp 24-29 Conference Location: Stuttgart, Germany; Conference Date: April 6-8, 2017

Piker D (2013) Kangaroo: form finding with computational physics. Archit Des 83(2):136-137

Prado M, Dörstelmann M, Schwinn T, Menges A, Knippers J (2014) Core-less filament winding. In: McGee W, Ponce M (eds) Robotic fabrication in architecture, art and design. Springer International Publishing, Cham, pp 275-289

Reichert S, Schwinn T, La Magna R, Waimer F, Knippers J, Menges A (2014) Fibrous structures: an integrative approach to design computation, simulation and fabrication for lightweight, glass and carbon fibre composite structures in architecture based on biomimetic design principles. Comput-Aided Des 52:27-39

Rutten D (2013) Galapagos: on the logic and limitations of generic solvers. Archit Des 83:132-135

Sarles A, Leo D, Riffle J. (2006) Improved composite rigidization via temperature-controlled resistive heating. In: 47th AIAA/ASME/ ASCE/AHS/ASC Structures, Structural Dynamics, and Materials Conference

SCI P358 (2014) Joints in steel construction: Simple joints to eurocode 3 (2014 reprint). https://www.steelconstruction.info/Simpl e_connections\#Costs. Accessed 18 May 2017

Solly J (2020) Virtual prototyping tools for a winding-based composite fabrication technique. UCL Press, London

Vasey L, Baharlou E, Dörstelmann M, Koslowski V, Prado M, Schieber G, Menges A, Knippers J (2015) Behavioral design and adaptive robotic fabrication of a fiber composite compression shell with pneumatic formwork. In: Combs L, Perry C (eds) Computational ecologies: design in the anthropocene, Proceedings of the 35th Annual Conference of the Association for Computer Aided Design in Architecture (ACADIA). University of Cincinnati, Cincinnati, pp 297-309

Yablonina M, Menges A (2018) Towards the development of fabrication machine species for filament materials. Robot Fabr Architect Art Des 2018:152-166

Publisher's Note Springer Nature remains neutral with regard to jurisdictional claims in published maps and institutional affiliations. 